\title{
Influência da temperatura da massa de grãos sobre a toxicidade do ozônio a Tribolium castaneum
}

\author{
Alexandre de M. Pereira ${ }^{1}$, Lêda R. D'A. Faroni ${ }^{1}$, Adalberto H. de Sousa ${ }^{2}$, Wilfredo I. U rruchi ${ }^{3}$ \& Juliana L. Paes ${ }^{1}$
}

\section{RESU M O}

Considerando que a temperatura da massa de grãos pode influenciar o período de exposição dos fumigantes, desenvolveu-se este trabalho com o propósito de investigar a influência de temperaturas crescentes da massa de grãos (20, 30,35 e $40^{\circ} \mathrm{C}$ ) no período de exposição do ozônio necessário para matar 50 e $95 \%$ de insetos adultos de Tribolium castaneum H erbst (Coleoptera: Tenebrionidae). Grãos de milho foram distribuídos em recipientes cilíndricos de PVC com conexões para injeção e exaustão de gás. 0 s insetos contidos em gaiolas foram colocados na camada mediana da massa de grãos em cada recipiente e estes, por sua vez foram fumigados com ozônio na concentração de $50 \mathrm{ppm}$. $0 \mathrm{~s}$ tempos letais $\left(\mathrm{TL}_{50} \mathrm{e} \mathrm{TL}_{95}\right.$ ) para os insetos adultos de $\mathrm{T}$. castaneum foram reduzidos com o aumento da temperatura da massa de grãos, de 20 para $30{ }^{\circ} \mathrm{C}$, porém a $35^{\circ} \mathrm{C}$ os tempos letais não foram reduzidos em relação às temperaturas menores, mas a $40^{\circ} \mathrm{C}$ os tempos letais foram reduzidos novamente, resultando no menor $\mathrm{TL}_{95}$.

Palavras-chave: ozonização, pragas de grãos, armazenamento

\section{Influence of the grain temperature on the ozone toxicity to Tribolium castaneum}

\begin{abstract}
Considering that the grain temperature may influence the exposure time of the fumigants, the present study was carried out to investigate the influence of increasing temperatures of the grain mass $\left(20,30,35\right.$ and $\left.40^{\circ} \mathrm{C}\right)$ on the exposure time to ozone required to kill 50 and $95 \%$ of adult insects of Tribolium castaneum (Herbst) (Coleoptera: Tenebrionidae). Maize grains were distributed in cylindrical polyvinyl chloride recipients having connections for gas injection and exhaustion. The insects held in cages were placed at the median height of the grain mass in each recipient, which was then fumigated with ozone at the concentration of $50 \mathrm{ppm}$. The lethal times $\left(\mathrm{LT}_{50}\right.$ and $\left.L T_{95}\right)$ for adult insects of $\mathrm{T}$. castaneum were reduced by increasing grain temperature, from 20 to $30^{\circ} \mathrm{C}$; however, at $35^{\circ} \mathrm{C}$ the lethal times were not reduced compared with lower temperatures; but at $40^{\circ} \mathrm{C}$ the exposure time decreased again, resulting in lower $\mathrm{LT}_{95}$.
\end{abstract}

Key words: ozonization, grain insect pests, storage

1 DEA/UFV. 36570-000, Viçosa, MG. Fone (31) 3899-1874. E-mail: Ifaroni@ufv.br

2 DBA/U FV. Fone (31) 3899-1919. E-mail: adal berto@insecta.ufv.br

3 Departamento de Física/ITA. Pça. Marechal Eduardo Gomes, 50, Vila das Acácias, CEP. 12228-900. São José dos Canpos, SP. Fone (12) 347-5940. E-mail: wiurruchi@yahoo.com.br 


\section{INTRODUÇÃO}

$\mathrm{O}$ ataque severo de insetos-praga durante $\mathrm{o}$ armazenamento de grãos é responsável por perdas consideráveis no setor de armazenamento de produtos agrícolas em todo o mundo. Dentre as espécies de insetos mais importantes, o gorgulho Tribolium castaneum Herbst (Coleoptera: Tenebrionidae) se destaca em virtude de infestar farinhas e grãos de cereais, além de ser uma das primeiras espécies a aparecer em produtos recentemente colhidos e armazenados (Loeck, 2002).

$\mathrm{O}$ controle desses insetos tem sido prioritário com o uso do inseticida fumigante fosfeto de alumínio, cujo princípio ativo é a fosfina, o que representa grande risco ao desenvolvimento de resistência a este produto, visto que o uso contínuo de determinado inseticida durante muito tempo, aliado a aplicações inadequadas, permite o aumento da pressão de seleção de indivíduos resistentes (McKenzie, 1996). Adicionalmente ao conhecimento de resistência, a preocupação dos consumidores quanto à qualidade dos alimentos, vem incentivando o desenvolvimento de novas técnicas de controle de insetos-praga de produtos armazenados.

Dentre as principais alternativas ao uso da fosfina está a atmosfera modificada, empregando-se o gás ozônio $\left(\mathrm{O}_{3}\right)$ como agente controlador, um forte oxidante com propriedades inseticidas já demonstradas para insetos-praga de grãos armazenados (Kells et al., 2001; Zhanggui et al., 2003); sua utilização na agricultura vem se tornando atrativa, pelo fato de poder ser gerado no próprio local de uso e de seu produto de degradação, o oxigênio $\left(\mathrm{O}_{2}\right)$, não deixar resíduo nos grãos (Mendez et al., 2003); no entanto, sabe-se pouco a respeito dos agentes abióticos que podem interferir na eficácia de sua aplicação.

A temperatura da massa de grãos se destaca por se tratar de um dos agentes determinantes do rítmo da atividade metabólica dos insetos, visto que temperaturas mais elevadas tornam os insetos mais suscetíveis à ação dos fumigantes (Faroni et al., 2002); conseqüentemente, o período de exposição necessário para se obter controle eficiente de insetos pode ser reduzido conhecendo-se a temperatura ideal de sua aplicação (White \& Leesch, 1996), o que maximiza a viabilidade econômica de um produto, porém é importante lembrar que temperaturas mais elevadas podem ser responsáveis pela degradação do inseticida durante sua aplicação (Pimentel et al., 2004, 2005).

Considerando que a temperatura da massa de grãos interfere na ação inseticida do ozônio durante o processo de sua aplicação, desenvolveu-se este trabalho com o propósito de investigar a influência da temperatura da massa de grãos no período de exposição do ozônio sobre a mortalidade de $T$. castaneum.

\section{MATERIAL E MÉTODOS}

Os experimentos foram realizados no Setor de Pré-Processamento e Armazenamento de Produtos Agrícolas do Departamento de Engenharia Agrícola da Universidade Federal de Viçosa (UFV). Investigou-se a influência de temperaturas crescentes da massa de grãos $(20,30,35$ e 40 $\left.{ }^{\circ} \mathrm{C}\right)$ sobre o período de exposição do ozônio letal para 50 e 95\% de insetos adultos de T. castaneum $\left(\mathrm{TL}_{50}\right.$ e $\mathrm{TL}_{95}$ ), utilizando-se a dose de 50 ppm de ozônio em uma vazão de $8 \mathrm{~L}$ $\min ^{-1}$.

\section{Acondicionamento dos grãos e contenção dos insetos}

Grãos de milho $(25 \mathrm{~kg})$ foram acondicionados em recipientes cilíndricos de PVC com 0,20 m de diâmetro e $1 \mathrm{~m}$ de altura e a $0,10 \mathrm{~m}$ do fundo de cada recipiente se colocou uma tela metálica para sustentação dos grãos e formação de uma câmara, para melhor distribuição do gás; já nas tampas inferior e superior dos cilindros foram instaladas conexões para injeção e exaustão do gás e no corpo do cilindro se inseriu um sensor para o monitoramento da temperatura da massa de grãos. Para manter os insetos na camada mediana da massa de grãos, 20 adultos de $T$. castaneum foram colocados em $0,330 \mathrm{~kg}$ de grãos de milho contidos em gaiolas circulares de PVC, com 0,03 m de altura e $0,15 \mathrm{~m}$ de diâmetro. $\mathrm{O}$ fundo e a tampa das gaiolas foram confeccionados com tecido de organza, de forma que permitisse a passagem do ozônio ou do ar atmosférico e evitasse a fuga dos insetos.

\section{Bioensaios de tempo-resposta}

Os períodos de exposição do ozônio necessários para matar o menor número de insetos (mortalidade semelhante à da testemunha) e os períodos de exposição em que a mortalidade dos insetos foi próxima de $100 \%$, foram estimados para todas as temperaturas da massa de grãos, por meio de testes preliminares. A partir dos valores obtidos nesses testes, os períodos de exposição intermediários foram definidos, por meio dos quais se estimaram os tempos letais para 50 e $95 \%$ de mortalidade $\left(\mathrm{TL}_{50}\right.$ e $\left.\mathrm{TL}_{95}\right)$.

Utilizaram-se, para as temperaturas de 20 e $30{ }^{\circ} \mathrm{C}$, os períodos de exposição de 24, 48, 72, 120 e $168 \mathrm{~h}$. Com relação às temperaturas de 35 e $40{ }^{\circ} \mathrm{C}$, os períodos de exposição empregados foram de 24, 48, 72, 96, 120 e $168 \mathrm{~h}$, enquanto a testemunha se constituía apenas de ar atmosférico $(78 \%$ de $\mathrm{N}_{2}, 0,03 \%$ de $\mathrm{CO}_{2}$ e $21 \%$ de $\mathrm{O}_{2}$ ); por fim, três repetições para cada temperatura e período de exposição foram utilizadas e a avaliação da mortalidade dos insetos feita 48 h após o término de cada tratamento.

\section{Controle da temperatura da massa de grãos}

Os cilindros contendo os grãos de milho foram acondicionados em câmaras climáticas cuja temperatura do ar foi controlada conforme a temperatura da massa de grãos que, por sua vez, foi monitorada através de um sistema computacional de aquisição e armazenamento de dados, denominado 1-wire ${ }^{\mathrm{TM}}$; este sistema se constitui de uma rede de transmissão de dados em que, através de um programa, um único cabo condutor permite a comunicação entre o computador e os sensores de temperatura na massa de grãos (Martins et al., 2004).

\section{Obtenção do ozônio e do ar atmosférico}

O gás ozônio foi obtido de um gerador desenvolvido pelo Departamento de Física do Instituto Tecnológico de Aeronáu- 
tica (ITA). No processo de geração do gás utilizou-se, como insumo, ar ambiente seco (ponto de orvalho menor que -40 ${ }^{\mathrm{E}} \% \mathrm{C}$ ), passando através de um reator refrigerado, no qual existe a Descarga por Barreira Dielétrica (DBD); esse tipo de descarga é produzido ao se aplicar alta voltagem entre dois eletrodos paralelos havendo, entre eles, um dielétrico (vidro) e um espaço livre por onde flui o ar seco ou o oxigênio; neste espaço livre é produzida uma descarga em forma de filamentos e gerados elétrons com energia suficiente para produzir a quebra das moléculas de oxigênio, formando o ozônio $\left(\mathrm{O}_{3}\right)$.

O ar seco utilizado como insumo foi obtido a partir de um compressor de ar instalado juntamente com um filtro de alumina, na saída do qual se instalou uma conexão com duas saídas: uma passando pelo gerador de ozônio e a outra indo diretamente para o sistema em que se utilizou ar ambiente (testemunha). A produção de ozônio foi regulada em função da tensão aplicada, ajustada por meio de um variador de tensão do gerador e do fluxo de ar seco, monitorado com auxílio de um medidor de fluxo de ar. A concentração de ozônio foi medida com um espectrofotômetro com precisão de $0,1 \mathrm{~g}$ $\mathrm{m}^{-3}$. Com o intuito de aumentar a precisão nas leituras de concentração de $\mathrm{O}_{3}$, conectou-se um multímetro ao espectrofotômetro para que fosse possível estabelecer uma relação entre os valores lidos no espectrofotômetro $\left(\mathrm{g} \mathrm{m}^{-3}\right)$ e a corrente elétrica $(\mathrm{mA})$.

\section{Análise dos dados}

Os dados de mortalidade foram corrigidos pela fórmula de Abbott (1925) e submetidos à análise de Probite, utilizando-se o procedimento PROC PROBIT do programa SAS ${ }^{\circledR}$ for Windows $^{\mathrm{TM}}$, versão 6.12 , para avaliação do efeito das diferentes temperaturas sobre o período de exposição gerando, assim, as curvas de mortalidade.

\section{RESULTADOS E DISCUSSÃO}

Os tempos letais para 50 e $95 \%$ dos insetos adultos de $T$. castaneum, submetidos a atmosfera modificada com $50 \mathrm{ppm}$ de ozônio, foram influenciados pela elevação da temperatura da massa de grãos e em relação às temperaturas de 20, 30,35 e $40{ }^{\circ} \mathrm{C}$ foram, respectivamente, de 71,39 e 151,85 ; 59,61 e 115,$62 ; 82,93$ e 138,22 e 69,52 e 105,12 h (Tabela 1).

Tabela 1. Toxicidade relativa de ozônio para adultos de Tribolium castaneum, a temperatura de $20,30,35$ e $40^{\circ} \mathrm{C}$ da massa de grãos

\begin{tabular}{cccccc}
\hline $\begin{array}{c}\mathbf{T} \\
\left({ }^{\circ} \mathbf{C}\right)\end{array}$ & $\begin{array}{c}\text { Incl. } \pm \\
\text { E.P.M. }\end{array}$ & $\mathrm{TL}_{50}$ (IC) & $\mathrm{TL}_{95}$ (IC) & $\chi^{2}$ & $\mathbf{P}$ \\
\hline 20 & $1,20 \pm$ & 71,39 & 151,85 & 5,87 & 0,12 \\
& 0,06 & $(64,60-78,84)$ & $(129,85-188,39)$ & & \\
30 & $1,20 \pm$ & 59,61 & 115,62 & 3,35 & 0,18 \\
& 0,02 & $(52,12-69,13)$ & $(94,83-157,49)$ & & \\
35 & $1,86 \pm$ & 82,93 & 138,22 & \multirow{2}{*}{4,81} & 0,18 \\
& 0,13 & $(77,78-88,23)$ & $(125,45-148,10)$ & & \\
40 & $1,50 \pm$ & 69,52 & 105,12 & 4,21 & 0,38 \\
& 0,20 & $(64,93-73,85)$ & $(94,59-127,29)$ & 4,21 & \\
\hline
\end{tabular}

T - temperatura, Incl. - inclinação, E.P.M. - erro padrão da média, TL tempo letal (h), IC - intervalo de confiança a $95 \%$ de probabilidade, $c^{2}$ qui-quadrado e $\mathrm{P}$ - probabilidade
O menor e o maior período de exposição para controlar $50 \%$ dos insetos foram de 59,61 e 82,93 h, respectivamente, quando os insetos foram submetidos a temperaturas da massa de grãos de 30 e $35{ }^{\circ} \mathrm{C}$; para controlar $95 \%$ dos insetos, esses períodos de exposição foram de 105,12 e 151,85 h, quando os insetos foram submetidos, respectivamente, a temperaturas da massa de grãos de 40 e $20{ }^{\circ} \mathrm{C}$ (Tabela 1).

Os tempos letais para 50 e $95 \%$ dos insetos foram reduzidos com a elevação da temperatura da massa de grãos, de 20 para $30{ }^{\circ} \mathrm{C}$, porém na temperatura subseqüente $35^{\circ} \mathrm{C}$, esses tempos letais não foram reduzidos, respondendo pelo maior $\mathrm{TL}_{50}$ e por elevação significante do $\mathrm{TL}_{95}$ em relação às temperaturas menores; já na temperatura de $40^{\circ} \mathrm{C}$, os tempos letais foram reduzidos novamente, proporcionando o menor $\mathrm{TL}_{95}$ (Tabela 1).

Para que a mortalidade dos primeiros insetos fosse computada, as temperaturas de 35 e $40{ }^{\circ} \mathrm{C}$ demandaram períodos de exposição semelhantes entre si e maiores que as temperaturas de 20 e $30{ }^{\circ} \mathrm{C}$ (Figura 1); contudo, para alcançar a mortalidade máxima de insetos as temperaturas de 35 e 40 ${ }^{\circ} \mathrm{C}$ responderam por um comportamento particular, indicando serem responsáveis, respectivamente, pelo maior e menor período de exposição do ozônio na massa de grãos.

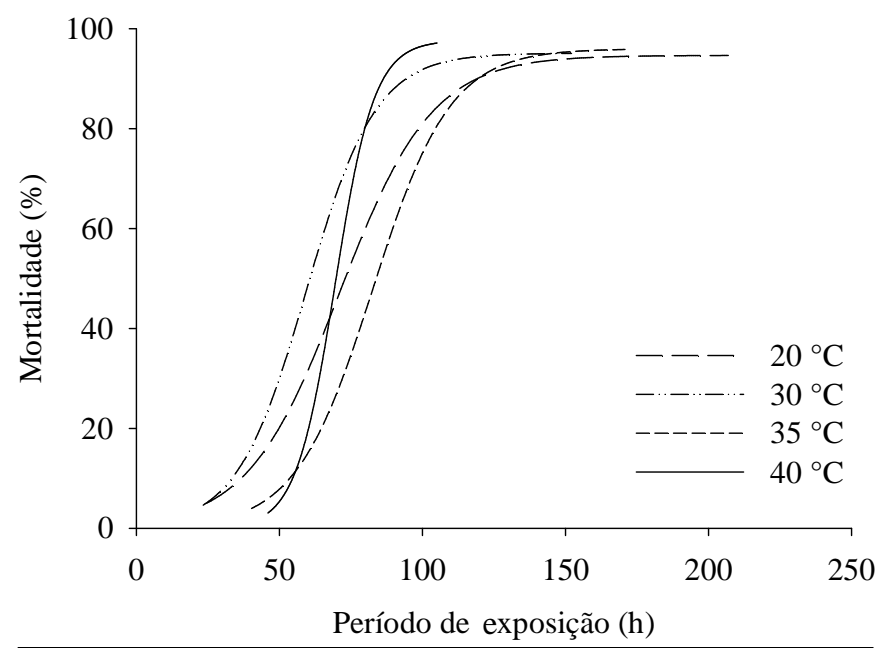

Figura 1. Mortalidade de Tribolium castaneum em diferentes temperaturas da massa de grãos submetidos a diferentes períodos de ozonização

Dentre os fatores que influenciam o período de exposição dos fumigantes, a temperatura da massa de grãos se destaca devido à sua relação direta com a suscetibilidade dos insetos (Throne et al., 2000) e por atuar como agente de degradação de inseticidas (Pimentel et al., 2005); neste contexto, se atribuem os resultados dos tempos letais obtidos nesta investigação, à interação entre suscetibilidade dos insetos e potencial tóxico do gás em cada temperatura.

Do ponto de vista biológico, o aumento da suscetibilidade dos insetos, em função da elevação da temperatura, se deve ao aumento de sua taxa respiratória (Throne et al., 2000), acompanhada de uma elevação da atividade metabólica (consumo de $\mathrm{O}_{2}$ e produção de $\mathrm{CO}_{2}$ ) (Celaro, 2002), o que pode resultar em desbalanço das trocas gasosas dos insetos, propiciando maior gasto energético para a manutenção da ho- 
meostase (Hostetler et al., 1994; Harak et al., 1999), que é o processo responsável pela manutenção da integridade do ambiente interno das células (Nation, 2002).

Admitindo-se que a elevação da temperatura tenha relação com o aumento da sensibilidade dos insetos, previa-se que sua suscetibilidade aumentasse conforme a elevação da temperatura da massa de grãos, mas se verificou que na temperatura de $35{ }^{\circ} \mathrm{C}$ os insetos exigiram maiores períodos de exposição que nas temperaturas de 20 e $30^{\circ} \mathrm{C}$, sugerindo que a temperatura da massa de grãos a $35^{\circ} \mathrm{C}$ pode ter interferido na manutenção da dose aplicada (50 ppm). Como o processo de degradação do ozônio principia na temperatura de $35^{\circ} \mathrm{C}$ (Delimpasis, 2006), é possível que o aumento do período de exposição nesta temperatura esteja relacionado a perdas na concentração do ozônio; isto pode ter contribuído para que as análises de regressão dos $T_{50}$ e $T L_{95} \mathrm{em}$ função da elevação da temperatura da massa de grãos, não apresentassem variação significativa $\left(\mathrm{TL}_{50}: \mathrm{y}=64,92+0,19 \mathrm{x}, \mathrm{F}=0,060 ; \mathrm{P}=0,83\right.$; $\mathrm{R}^{2}=0,029$ e TL $95: \mathrm{y}=198,27-2,36 \mathrm{x}, \mathrm{F}=3,18 ; \mathrm{P}=0,22 ; \mathrm{R}^{2}=$ 0,61 ; em que y é tempo letal e $\mathrm{x}$ é temperatura).

O fato dos insetos submetidos a temperatura de $40{ }^{\circ} \mathrm{C}$ apresentarem menores tempos letais que aqueles submetidos a temperatura de $35{ }^{\circ} \mathrm{C}$, deve estar associado à sua maior sensibilidade na temperatura de $40{ }^{\circ} \mathrm{C}$ e não a $35^{\circ} \mathrm{C}$, pois esta é a temperatura ótima para o desenvolvimento do $T$. castaneum (Rees, 1996). Soderstrom et al. (1992) também constataram influência da temperatura sobre o período de exposição de $T$. castneum a atmosferas modificadas e, ainda, aumento da mortalidade de larvas quando as temperaturas foram iguais ou superiores a $38{ }^{\circ} \mathrm{C}$, combinadas com atmosferas enriquecidas com $\mathrm{CO}_{2}$ ou deficientes em $\mathrm{O}_{2}$.

É provável que o maior período de exposição necessário para que se registrassem os primeiros insetos mortos nas temperaturas de 35 e $40{ }^{\circ} \mathrm{C}$, tenha relação com os resultados encontrados por Kells et al. (2001); esses autores constataram que o processo de fumigação com ozônio apresenta duas fases distintas, em que na primeira ocorre rápida degradação do ozônio e movimentação lenta pela massa de grãos e, na segunda, o ozônio flui livremente através da massa de grãos com pouca degradação, haja vista que nesse momento os locais responsáveis pela degradação do ozônio ficam saturados; assim, é possível que a menor eficácia observada nas primeiras horas de exposição do ozônio (Figura 1), nas temperaturas de 35 e $40{ }^{\circ} \mathrm{C}$, se deva à degradação natural que ocorre nas primeiras horas, referentes à primeira fase, somada à degradação comum do ozônio nessas temperaturas (Delimpasis, 2006).

\section{CONCLUSÕES}

1. A temperatura da massa dos grãos de milho durante a ozonização influenciou a toxicidade do ozônio em insetos adultos de $T$. castaneum.

2. Os tempos letais encontrados para $95 \%$ dos insetos mostraram que a menor toxicidade do ozônio ocorreu na temperatura da massa de grãos de $20{ }^{\circ} \mathrm{C}$ e as temperaturas de 30 e $40{ }^{\circ} \mathrm{C}$ causaram maior toxicidade do ozônio.
3. Os resultados desta investigação indicam que o ozônio poderá tornar-se uma das principais alternativas ao uso dos inseticidas convencionais em regiões de clima tropical, uma vez que este gás apresentou alta toxicidade para os insetos nas temperaturas estudadas.

\section{AGRADECIMENTOS}

Este trabalho faz parte do projeto Avaliação do uso de ozônio como fumigante no controle de insetos-praga de grãos armazenados, financiado pelo Conselho Nacional de Desenvolvimento Científico e Tecnológico - CNPq e pelo Instituto Tecnológico da Aeronáutica - ITA, aos quais os autores agradecem.

\section{LITERATURA CITADA}

Abbott, W. S. A. A method of computing the effectiveness of an insecticide. Journal of Economic Entomology, v.18, n.1, p.265-267, 1925.

Celaro, J. C. Métodos curativos de controle de pragas de grãos armazenados. In: Lorini, I.; Miike, L. H.; Scussel, V. M. Armazenagem de grãos. Campinas: IBG, 2002. cap. 7.2, p.493-529.

Delimpasis, K. Ozone application for wastewater color removal. Public Service Ads by Google (online). http://www.etelescope.gr/en/cat04/art04_010601.htm. 22 Fev. 2006.

Faroni, L. R. D'A.; Guedes, R. N. C.; Berbeert, P. A.; Silva, A. P. R. A. Atmosfera modificada no controle das pragas de grãos armazenados. In: Lorini, I.; Miike, L. H.; Scussel, V. M. (ed.) Armazenagem de grãos. Campinas: IBG, 2002. cap. 7.4, p.463-491.

Harak, M.; Lamprecht, I.; Kuusik, A.; Hiiesaar, K.; Metspalu, L.; Tartes, U. Calorimetric investigations of insect metabolism and development under the influence of a toxic plant extract. Thermochemistry Acta, v.333, n.1, p.39-48, 1999.

Hostetler, M. E.; Anderson, J. F.; Lanciani, C. Pesticide resistance and metabolic rate in german cockroach (Dictyoptera: Blattellidae). Florida Entomologist, v.77, n.2, p.288-290, 1994.

Kells, S. A.; Mason, L. J.; Maier, D. E.; Wolososhuk, C. P. Efficacy and fumigation characteristics of ozone in stored maize. Journal of Stored Products Research, v.37, n.4, p.371-383, 2001.

Loeck, A. E. Principais pragas que atacam produtos armazenados. In: Loeck, A. E. (ed.) Pragas de produtos armazenados. Pelotas: EGUFPEL, 2002. cap. 7, p.35-59.

Martins, J. H.; Monteiro, P. M. B.; Mota, A. M. N.; Fonseca, J. A. G. The 1-wire ${ }^{T M}$ System - An Application for Agricultural Processes. In: Portuguese Conference on Automatic Control, 6, 2004, Faro. Proceedings... Faro: Controlo, v.2, p.602-607, 2004.

McKenzie, J. A. Selection against resistant phenotypes. In: McKenzie, J. A. (ed.) Ecological and evolutionary aspects of insecticide resistance. Austin: Academic Press, 1996. cap. 4, p.89-121.

Mendez, F.; Maier, D. E.; Mason, L. J.; Woloshuk, C. P. Penetration of ozone into columns of stored grains and effects on chemical composition and performance. Journal of Stored Products Research, v.39, n.1, p.33-44, 2003. 
Nation, J. L. Insect physiology and biochemistry. Boca Raton: CRC Press LLC, 2002. p.371-380.

Pimentel, M. A. G.; Faroni, L. R. D’A.; Gonçalves, J. R.; Oliveira, C. R. F.; Alencar, E. R. Influencia da temperatura na eficácia biológica de pirimifós metílico em milho armazenado. Revista Brasileira de Armazenamento, v.29, n.1, p.5861, 2004.

Pimentel, M. A. G.; Faroni, L. R. D’A.; Guedes, R. N. C.; Gonçalves, J. R.; Oliveira, C. R. F. Eficácia biológica de bifentrina aplicado em milho armazenado sob diferentes temperaturas. Revista Brasileira de Engenharia Agrícola e Ambiental, v.9, n.2, p.263-267, 2005.

Rees, D. P. Coleoptera. In: Subramanyam, B.; Hagstrum, D. W. (ed.) Integrated management of insects in stored products. New York: Marcel Dekker, 1996. cap. 1, p.1-39.
Soderstrom, E. L.; Brandl, D. G.; Mackey, B. High temperature combined with carbon dioxide enriched or reduced oxygen atmospheres for control of Tribolium castaneum (Herbst) (Coleoptera: Tenebrionidae). Journal of Stored Products Research, v.28, n.4, p.235-238, 1992.

Throne, J. E.; Baker, J. E.; Messina, F. J.; Kramer, K. J.; Howard, J. A. Varieal resistance. In: Subramanyam, B.; Hagstrum, D. W. (ed.) Alternatives to pesticides in stored-product IMP. Massachusetts: Kluwer Academic, 2000. cap. 7, p.165-192.

White, N. D. G.; Leesch, J. G. Chemical control. In: Subramanyam, B.; Hagstrum, D. W. (ed.) Integrated management of insects in stored products. New York: Marcel Dekker, 1996. cap. 7, p.287-330.

Zhanggui, Q.; Xia, W.; Gang, D.; Xiaoping, Y.; Xuechao, H.; Deke, $\mathrm{X}$.; Xingwen, L. Investigation of the use of ozone fumigation to control several species of stored grain insects. In: International Working Conference on Stored-Product Protection, 8, 2003, York. Advances in stored product protection, York: 2003, p.846-851. 